\title{
REGISTRO DE PLAGIOMETRIONA CLAVATA (FABRICIUS 1798) (COLEOPTERA: CHRYSOMELIDAE) EN CHILE SILVESTRE CAPSICUM ANNUUM, DE BAJA CALIFORNIA SUR, MÉXICO
}

Tejas, R. A., R. Servín, A. Nieto-Garibay \& A. Marín. 2011. Registro de Plagiometriona clavata (Fabricius 1798) (Coleoptera: Chrysomelidae) en chile silvestre Capsicum annuum, de Baja California Sur, México. Acta Zool. Mex. (n. s.), 27(1): 201-205.

Tejas, R. A., R. Servín, A. Nieto-Garibay \& A. Marín. 2011. Record of Plagiometriona clavata (Fabricius 1798) (Coleoptera: Chrysomelidae) on wild chili Capsicum annuum, of Baja California Sur, Mexico. Acta Zool. Mex. (n. s.), 27(1): 201-205.

ABSTRACT. Plagiometriona clavata Fabricius, 1798 is reported for the first time on Capsicum annuum var. aviculare, considerated like the wild progenitor of the cultivated chiles.

Los crisomélidos son insectos que se alimentan de hojas, tallos y raíces de plantas, esta familia incluye especies de importancia agrícola que pueden generar cuantiosas pérdidas económicas. Por su diversidad morfológica y adaptativa se han generado varias sinonimias entre las especies, tal situación ha involucrando a los taxónomos especialistas de dichos insectos. Uno de los trabajos más recientes realizados en México, es el de Andrews y Gilbert (2005), ellos revisaron una colección de 16,000 especímenes y refieren para la Península de Baja California, 120 especies no descritas, de las cuales 29 son endémicas y 91 son consideradas como nuevos registros para esta región. Varios de los insectos estudiados pertenecen a Cassidinae, en donde se incluye Plagiometriona clavata, que tiene como sinónimos a Coptocycla clavata (Fabricius 1798), Boheman; Deloyala clavata (Fabricius), Dejean 1837 y Cassida clavata Fabricius 1798 (Woodruff 2006), y se conoce comúnmente como tortuga de tierra. Los primeros datos de su distribución fueron proporcionados por Barber (1918), citándola en quince estados de Estados Unidos de América (EUA) sobre varios hospederos. Pa-

Recibido: 24/05/2010; aceptado: 09/09/2010. 
checo (1986) cita a P. clavata como plaga potencial en la Meseta Central de México, debido a que tanto adultos como inmaduros se alimentan del follaje de plantas de chile, lo que limita el rendimiento del cultivo. Trabajos recientes amplían su distribución en varias regiones de EUA y el Norte de México (Arnett 2000), en donde se incluye Baja California Sur (Andrews y Gilbert 2005).

Al igual que otros crisomélidos Cassidinae, $P$. clavata utiliza sus heces y exuvias como barreras de protección, las hembras depositan su excremento en sus huevos lo que según algunos autores, provee un repelente químico eficiente en contra de parasitoides y depredadores (Barrows 1979, Olmstead 1994, Nogueira y Trigo 2002). Las larvas de manera similar pueden cargar masas de exuvias y heces en el último segmento abdominal formando una estructura en forma de placa (Eisner y Eisner 2000). Vencl y colaboradores (1999), demostraron que estas placas particularmente en $P$. clavata, pueden contener metabolitos como saponinas esteroidales, ácidos grasos y otros compuestos que constituyen una barrera en contra del ataque de hormigas. Con respecto a los hospederos, $P$. clavata es una especie polífaga con marcada preferencia por algunas solanáceas (Buzzi 1994).

Una especie vegetal de gran interés para el Centro de Investigaciones Biológicas del Noroeste S. C. (CIBNOR), es el chile piquín silvestre Capsicum annuum L. var. aviculare el cual se distribuye desde la parte sur de EUA, casi todo México, Centro y Suramérica. Esta planta se encuentra asociada con diferentes tipos de vegetación de ambientes áridos sobre lechos de ríos o arroyos (Medina et al. 2006). Parte de su importancia científica, estriba en que es considerada como el progenitor silvestre de las especies de chile domesticadas (Eshbaugh 1980) y se encuentra desde Sonora hasta Chiapas y de Tamaulipas hasta Yucatán, lo que le ha conferido amplia variación genética y diversos usos (Hernández-Verdugo et al. 2001). Por lo anterior, varios investigadores del CIBNOR y de otras instituciones realizan proyectos asociados con chile silvestre para evaluar su germinación, propagación por cultivo de tejidos, aclimatación y trasplante, así como su caracterización genética para el establecimiento de plantaciones que permitan una actividad económica sustentable a los productores interesados (Hernández-Verdugo et al. 2001, Alcaraz-Melendez et al. 2006, 2007).

Para apoyar tales proyectos y con objeto de aportar conocimiento sobre la entomofauna asociada con C. annuum var. aviculare, se realizaron observaciones estacionales durante 2007 y 2008 de varios ejemplares de chile silvestre, presentes en una comunidad vegetal de tipo selva baja caducifolia combinada con matorral xerófilo (Wiggins 1980), ubicada en el oasis de San Bartolo, con clima de tipo BWhw (e), con una temperatura media de $26.8^{\circ} \mathrm{C}$ y una precipitación pluvial de $320.3 \mathrm{~mm}$ (Arriaga y Rodríguez 1997). Dichas observaciones tuvieron como propósito determinar los insectos herbívoros presentes y el posible daño generado por ellos (Fig. 1), asimismo, se revisaron las especies vegetales asociadas con $C$. annuum var. aviculare para identificar otros hospederos de los insectos estudiados. El material entomológico ob- 


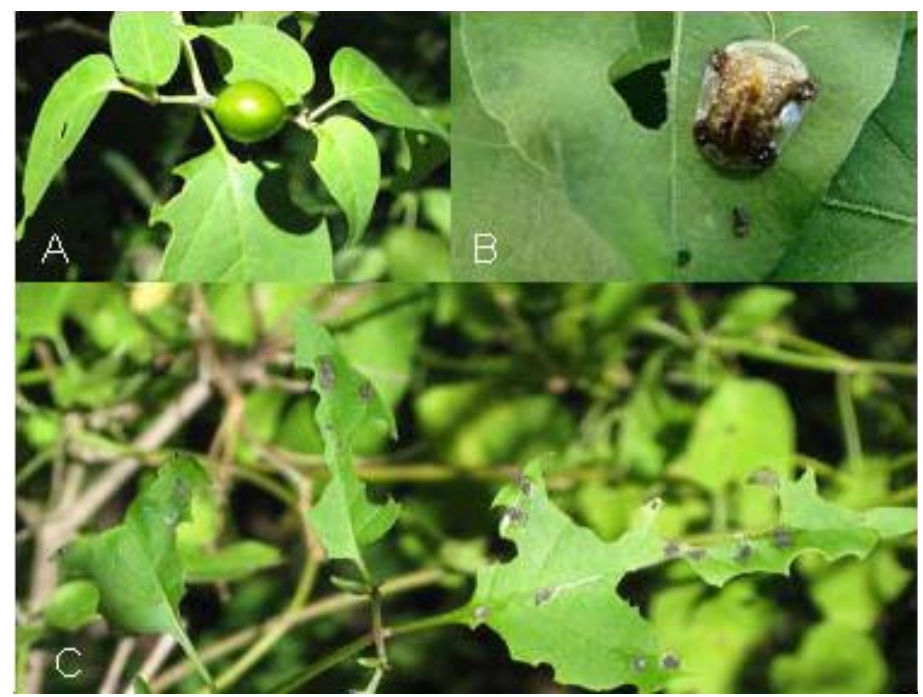

Figura 1. A) Capsicum annuum var. aviculare con fruto proveniente de San Bartolo, B.C.S. B) Plagiometriona clavata en follaje. C) Daño generado por P. clavata en C. annuum var. aviculare.

tenido se depositó en frascos con alcohol al 70\% y, las muestras vegetales colectadas para la obtención de formas inmaduras se depositaron en bolsas de papel. En el laboratorio de Entomología Agrícola con la ayuda de una lupa estereoscópica se prepararon e identificaron los insectos obtenidos, lo que fue corroborado por especialistas del Colegio de Postgraduados, asimismo, las plantas de chile fueron en su momento identificadas por especialistas del CIBNOR.

Los insectos obtenidos en C. annuum var. aviculare, fueron $P$. clavata (Fig. 1 A y B), siendo el único hospedero identificado en el área de estudio. Al respecto, se han citado otras especies vegetales para este insecto en México, como; papa, frijol y ajonjolí, de acuerdo con la colección de referencia del INIFAP. Con referencia a la abundancia poblacional de $P$. clavata en chile silvestre, se encontró una proporción máxima de 10 individuos por planta considerando tanto formas inmaduras como adultos, sin embargo, hubo plantas con un nivel de defoliación importante (Fig. 1 C). $\mathrm{Al}$ alimentarse solamente del follaje del chile silvestre, las poblaciones del insecto más elevadas se encuentran posteriores a las lluvias de verano, periodo en el que la planta desarrolla hojas. Durante el invierno y primavera, en cambio, dichos insectos se hayan ausentes debido a la falta de follaje en el chile silvestre. El hecho de encontrar a $P$. clavata solamente en ejemplares silvestres de $C$. annuum var. aviculare, sugiere que éste podría ser uno de los hospederos originales del insecto, dadas las condiciones insulares de Baja California Sur y el número de endemismos presentes 
Tejas et al.: Plagiometriona clavata en chile Capsicum annuum

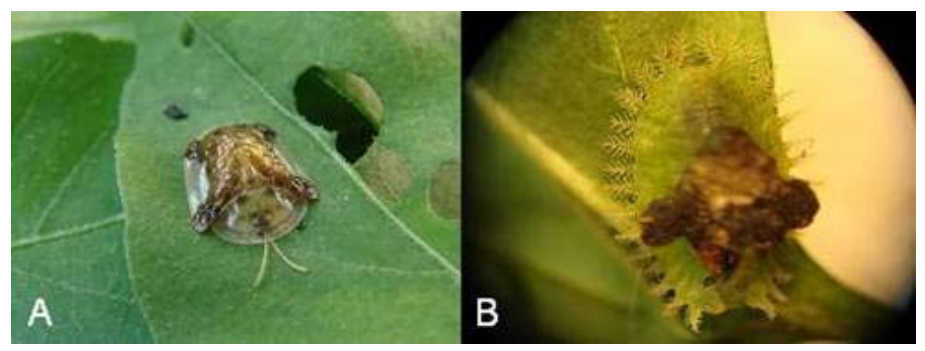

Figura 2. Plagiometriona clavata. A) Adulto alimentándose de hojas de Capsicum annuum var. aviculare. B) Larva con excretas en la región dorsal, utilizado como escudo de protección contra depredadores.

en los oasis de esta región (Arriaga y Rodríguez 1997). De acuerdo con lo anterior, es conveniente antes de establecer plantaciones de chile silvestre en la entidad, conocer diversos aspectos de la biología de $P$. clavata, para contar de forma preventiva con estrategias de manejo sustentable para el combate de este insecto y, evitar en lo posible el uso de insecticidas como ya ha sucedido en plantaciones comerciales de otros estados de México.

\section{LITERATURA CITADA}

Alcaraz-Meléndez, L., M. Rodríguez-Álvarez y S. Real-Cosío. 2006. Propagación por cultivo de tejidos de tres ecotipos de chiltepín (Capsicum annuum var. aviculare) como alternativa para cultivarlos en zonas semi-áridas. V Simposium Internacional sobre la Flora Silvestre de Zonas Áridas. 29-31 de marzo.

Alcaraz-Meléndez, L., M. Rodríguez-Álvarez y S. Real-Cosío. 2007. Evaluación en la etapa de aclimatación y trasplante al campo de plantas por cultivo in Vitro de chile chiltepín (Capsicum annuum var. aviculare) como alternativa para las zonas semi-áridas. Cuarta Convención Mundial de Chile. Tampico, Tamaulipas México. 15-17 de Julio.

Andrews, F.G. y A.J. Gilbert. 2005. A preliminary annotated checklist and evaluation of the diversity of the Chrysomelidae (Coleoptera) of the Baja California peninsula, Mexico. Insecta Mundi, 19: 89-116.

Arnett, Jr. R.H. 2000. American Insects: A handbook of the Insects of America North of Mexico. CRC Press. Boca Raton.

Arriaga, L. y R. Rodríguez E. 1997. Los oasis de la península de Baja California. 292 pp.

Barber H.S. 1918. A review of North American tortoise beetles (Chrysomelidae: Cassidinae). Proceedings of the Entomological Society of Washington, 18: 113-127.

Barrows E.M. 1979. Life cycles, mating, and color change in tortoise beetles (Coleoptera: Chrysomelidae: Cassidinae). The Coleopterists Bulletin, 33: 9-16.

Buzzi, Z.J. 1994. Host plants of Neotropical Cassidinae. In: P.H. Jolivet, M.L. Cox y E. Petitpierre. Novel aspects of the biology of Chrysomelidae. Kluver Academic Publ. Dordrecht, Boston, London. pp. 205-212.

Eisner, T. y M. Eisner. 2000. Defensive use of a fecal thatch by a beetle larva (Hemisphaerota cyanea). Proceedings of the National Academy of Sciences. USA, 97: 2632-2636. 
Eshbaugh W.H. 1980. The taxonomy of the genus Capsicum (Solanacea). Phytologia, 47: 153-166.

Hernández-Verdugo, S., R. Luna-Reyes y K. Oyama. 2001. Genetic structure and differentiation of wild and domesticated populations of Capsicum annuum (Solanacea) from Mexico. Plant Systematics and Evolution, 226: 129-142.

Nogueira-de-Sá, F. y J.R, Trigo. 2002. Do fecal shields provide physical protection to larvae of the tortoise beetles Plagiometriona flavescens and Stolas chalybea natural enemies. Entomologia Experimentalis et Applicata, 104: 203-206.

Medina, M.T., L.A. Rodríguez del Bosque, H. Villalón, O. Pozo, M. Ramírez, R. López, M. Lara, G. Gaona, A. Cardona \& A. Mora. 2006. El chile piquín (Capsicum annuum L. var. aviculare) en el noreste de México. Aspectos ecológicos y socioeconómicos. (En línea) Tu Revista Digi. U@, $T$ Julio-Septiembre 1: $11 \mathrm{pp}$.

Olmstead, K. 1994. Waste products as chrysomelid defenses. Pp. 311-318. In: P.H. Jolivet, M.L. Cox, y Petitpierre (Eds.), Novel aspects of the biology of Chrysomelidae. Kluwer Academic Publishers, Dordrecht.

Pacheco M.F. 1986. Plagas de los cultivos agrícolas en Sonora y Baja California. Libro Tec. No. 1. Centro de Investigaciones Agrícolas del Noroeste-CIANO.

Vencl, V.F., T.C. Morton, R.O. Mumma, y J.C. Schultz. 1999. Shield defense of a larval tortoise beetle. Journal of Chemical Ecology, 25. 549-566.

Wiggins, I.L. 1980. Flora of Baja California. Stanford University Press. Palo Alto. California.

Woodruff, R.E. 2006. Clavate tortoise beetle, Plagiometriona clavata (Fabricius) Insecta: Coleoptera: Chrysomelidae). University of Florida. IFAS Extension. 3pp.

AGRADECIMIENTOS. Al M.C. Margarito Rodríguez autor de las fotografías de C. annuum var. aviculare, a la M.C. Carmen Mercado por su apoyo en la obtención de una parte del material estudiado, al Sr. Bruno Granados por su colaboración en la toma de las fotografías de P. clavata y al Sr. Amado Cota por su participación en las salidas de campo.

\section{Tejas, R. A., R. Servín, A. Nieto-Garibay y A. Marín}

Centro de Investigaciones Biológicas del Noroeste, S.C.

Mar Bermejo \# 195, Col. Playa Palo de Santa Rita, La Paz, B.C.S., México CP 23090

Corresponding author: Rosalía Servín Villegas. E mail: rservin04@cibnor.mx. 